\title{
Efficacy and safety of Yinxing Mihuan oral solution in the treatment of patients with post-PCl chest pain: A multicenter, prospective, randomized, double-blind, placebo-controlled study
}

\section{Danping Xu}

Guangdong Provincial Hospital of Traditional Chinese Medicine

\section{Bingxin Wu}

2nd Clinical Medical College of Guangzhou University of Chinese Medicine: Guangdong Provincial Hospital of Traditional Chinese Medicine

\section{Zikai Yu}

China Academy of Chinese Medical Sciences Xiyuan Hospital

Jinhai Lin

2nd Clinical Medical College of Guangzhou University of Chinese Medicine: Guangdong Provincial Hospital of Traditional Chinese Medicine

\section{Haiyan Xu}

Chinese Academy of Medical Sciences \&amp; Peking Union Medical College Fuwai Hospital

\section{Luoqi Lin}

2nd Clinical Medical College of Guangzhou University of Chinese Medicine: Guangdong Provincial Hospital of Traditional Chinese Medicine

\section{Qingrong Liu}

Chinese Academy of Medical Sciences \&amp; Peking Union Medical College Fuwai Hospital

\section{Biying Zhong}

2nd Clinical Medical College of Guangzhou University of Chinese Medicine: Guangdong Provincial Hospital of Traditional Chinese Medicine

\section{Yining Ding}

2nd Clinical Medical College of Guangzhou University of Chinese Medicine: Guangdong Provincial Hospital of Traditional Chinese Medicine

\section{Zhiwei Huang}

2nd Clinical Medical College of Guangzhou University of Chinese Medicine: Guangdong Provincial Hospital of Traditional Chinese Medicine

\section{Miaoyang Lin}

2nd Clinical Medical College of Guangzhou University of Chinese Medicine: Guangdong Provincial Hospital of Traditional Chinese Medicine 
National Center for Cardiovascular Diseases China: Chinese Academy of Medical Sciences \&amp; Peking Union Medical College Fuwai Hospital

\section{Research Article}

Keywords: Coronary heart disease, chest pain, Percutaneous coronary intervention, anxiety, depression, Traditional Chinese medicine, Randomized controlled trial

Posted Date: April 12th, 2021

DOl: https://doi.org/10.21203/rs.3.rs-235622/v1

License: (c) (i) This work is licensed under a Creative Commons Attribution 4.0 International License. Read Full License 


\section{Abstract}

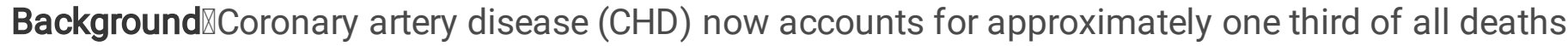
globally and up to one-quarter of patients may have either continues or recurrent post-PCl chest pain. Yinxing Mihuan oral solution (YMOS) has been proven to bring clinical benefits for patients with $\mathrm{CHD}$ and Ginkgo biloba extract can relieve depression in mice. No high-quality randomized controlled trials on YMOS have been published. The aim of this study is to evaluate the efficacy and safety of Yinxing Mihuan oral solution (YMOS) in the treatment of patients with post-percutaneous coronary intervention (PCl) chest pain. This study also aimed to relieve the symptoms of chest pain as a primary outcome, improve the anxiety as a secondary outcome, assess therapeutic effects of YMOS on chest pain, and explore its effects on chest pain caused by psychological disorders after PCl.

Methods: 320 patients with symptoms related to heart and a confirmed history of $\mathrm{PCl}$ for coronary heart disease (CHD) without the need to undergo selective coronary revascularization plan were recruited in this randomized controlled trial. The included participants were randomly assigned (at a ratio of 1:1) to experimental group and control group; patients in the experimental group received YMOS and standardized medical treatment, while those in control received placebo $(10 \mathrm{ml})$ for three times/day for 12 weeks and standardized medical treatment regimen. The endpoints of the study included Seattle angina questionnaire (SAQ) score, Hamilton anxiety rating scale (HAMA), Hamilton rating scale for depression (HRSD), Canadian Cardiovascular Society (CCS) angina score, New York Heart Association (NYHA) classification, visual analogue scale (VAS), 12-Item short form survey (SF-12), frequency of angina-related symptoms per week, nitroglycerin discontinuation, and reduction rate of HAMA score.

Discussion: This clinical trial can provide reliable evidence regarding the efficacy and safety of YMOS for patients with post-PCl chest pain.

Trial registration: This research has been registered at the Chinese Clinical Trial Registry (Registration No. ChiCTR2000028760) on January 1, 2020.

http://www.chictr.org.cn/showproj.aspx?proj=47528

\section{Background}

At present, coronary artery disease (CHD) accounts for approximately one third of all deaths worldwide, and the total number of deaths due to $\mathrm{CHD}$ is still increasing in China [1,2]. Managing patients with $\mathrm{CHD}$ has been substantially improved, and since Gruntzig's first angioplasty, percutaneous coronary intervention $(\mathrm{PCl})$ has become one of the most frequently performed therapeutic interventions $[3,4]$. However, complications associated with PCl still exist [5]. Up to one-quarter of patients may have either continues or recurrent post-PCl chest pain (PPCP)[6]. When restenosis or stent thrombosis is excluded, the possible causes of PPCP are as follows: drug-eluting stents (DESs), acetylcholine, microvascular dysfunction or coronary endothelial dysfunction, anxiety, and depression [7-11]. Chest pain and depression typically coexist, and $31-45 \%$ of patients with $\mathrm{CHD}$ suffer from clinically significant 
depressive symptoms $[12,13]$. A randomized controlled trial that involved 5158 patients with CHD revealed that depression is associated with angina pectoris rather than the severity of $\mathrm{CHD}$ [10]. Therefore, it is of great significance to alleviate the chest pain of PPCP patients, while improving their anxiety and depression symptoms.

Yinxing Mihuan oral solution (YMOS) is a chemical compound that is composed of Ginkgo biloba extract and Armillaria mellea mycelia extract powder. In clinical practice, it is mainly used for treating CHD, angina, and ischemic cerebrovascular disease, improving the symptoms of ischemic heart and brain, as well as anxiety and depression after ischemia. Three Chinese early-stage small sample-sized clinical studies [14-16] have shown that after treatment of chronic stable angina pectoris with YMOS alone for 4 weeks, the effective rate (clinical symptoms basically disappeared, blood pressure, heart rate, and blood lipid all returned to normal, and heart function and ST segment on an electrocardiogram (ECG) returned to normal) is within $16.7-57.5 \%$, which is higher than that of conventional Western medicine (antiischemic treatment) group (42.5\%) ( $P \otimes 0.05)$. After two weeks of combined treatment with enteric-coated aspirin tablets and clopidogrel tablets, the effective rate is $58.3 \%(35 / 60)$, which is $40 \%$ higher than that of enteric-coated aspirin tablets and clopidogrel tablets alone $(P<0.05)$. No adverse reactions were reported during the three small sample-sized clinical studies. However, according to the literature, there was a case of allergic skin pruritus caused by the medicine for the first time [17].

In addition, a previous study [18] showed that Ginkgo biloba extract can relieve depression in mice; several clinical trials [19-21] reported that Ginkgo biloba extract can particularly relieve anxiety symptoms, and its improvement effect is similar to that of benzodiazepines. However, this effect needs to be further confirmed by a larger sample-sized and more rigorous clinical research. Simultaneously, Armillaria, a genus of parasitic fungi, has been reported to be used for mental disorders, such as neurasthenia and insomnia [22].

To date, no multicenter, large sample clinical study has concentrated on the treatment of chest pain after $\mathrm{PCl}$ with YMOS. Based on the clinical study of pharmacodynamics and components of YMOS, the present clinical trial aimed to study the treatment of chest pain after $\mathrm{PCl}$ with YMOS, and assess the therapeutic effects on chest pain using disappearance rate of angina pectoris at the 12th week as the primary outcome, and simultaneously explore its efficacy and safety for chest pain caused by psychological disorders after $\mathrm{PCl}$.

\section{Methods}

In the current study, PPCP patients with CHD from Guangdong Provincial Hospital of Traditional Chinese Medicine (Guangzhou, China) were randomly assigned to experimental group and control; patients in experimental group received YMOS and standardized medical treatment, while those in control group received the placebo for three times/day for 12 weeks and standardized medical treatment for 12 weeks. Once the patients were assigned to the experimental group, they received laboratory tests and completed Seattle angina questionnaire (SAQ) score (physical limitation (PL), angina stability (AS), angina 
frequency (AF), treatment satisfaction (TS), and disease perception (DP), Hamilton anxiety rating scale (HAMA), Hamilton rating scale for depression (HRSD), Canadian Cardiovascular Society (CCS) angina score, New York Heart Association (NYHA) classification, visual analogue scale (VAS), and 12-Item short form survey (SF-12), while the frequency of angina-associated symptoms per week and the number of nitroglycerin use were recorded. The results of laboratory tests were used to evaluate the safety of YMOS, including blood routine examination, urine and stool routine examination, hepatic and renal function, fasting blood glucose and blood lipid, coagulation, troponin I (Tnl), ECG, and thyroid function test as required. The patients were followed-up for 8 weeks with repeated questionnaires every 4 weeks. At the beginning of the study, all the patients signed the written informed consent forms, in which they were informed about the risks and benefits of the treatment strategy. The study was guided by the Standard Protocol Items: Recommendations for Interventional Trials (SPIRIT) (Fig. 1). A flowchart of the trial is shown in Fig. 1.

\section{Inclusion and exclusion criteria}

The inclusion criteria were as follows: (1) Male or female patients who aged 18-85 years old; (2) Patients with a confirmed history of $\mathrm{PCl}$ of $\mathrm{CHD}$ within more than 30 days and less than 180 days, without need of selective coronary revascularization (coronary artery bypass grafting) plan assessed by cardiovascular specialists; (3) Patients with symptoms related to heart, such as chest distress, chest pain, palpitation, shoulder and back pain, shortness of breath, weakness, activity intolerance. Angina attack frequency (AF) dimension score in the SAQ would be < 80; (4) HAMA score would be in the range of 7-21 (mild-tomoderate anxiety); (5) Signing the written informed consent form.

The exclusion criteria were as follows: (1) Women who were pregnant or preparing to be pregnant or nursing; (2) Women who were at child-bearing age, while did not agree to use contraception on medication; (3) Patients with severe anxiety (HAMA score > 21); (4) Patients with major depression (HAMD score $\geq$ to 24); (5) Patients who were taking anti-anxiety drugs (e.g., selective serotonin reuptake inhibitors, selective serotonin and norepinephrine reuptake inhibitors, benzodiazepines, etc.); (6) Stable angina with grade 4 based on CCS; (7) Uncontrolled hypertension (systolic pressure $>180 \mathrm{mmHg}$ or diastolic pressure $>100 \mathrm{mmHg}$ ) despite ongoing antihypertensive treatment; (8) Heart failure with grade III or IV in NYHA; (9) Patients with CHD with atrial fibrillation; (10) Clinically significant complications, including liver dysfunction (alanine aminotransferase (ALT) or aspartate aminotransferase (AST) level more than 2 times higher than normal), renal dysfunction (serum creatinine level more than 2 times higher than normal), severe cardiopulmonary dysfunction, pulmonary hypertension, chronic obstructive pulmonary disease, history of cerebral hemorrhage or epilepsy, with need of anticonvulsant drugs; (11) Patients who did not take drug to treat CHD, while disappearance of related symptoms was noted at within 7 days of introduction period; (12) Patients with myocardial infarction or grade IV angina at 3 months before commencing the study; (13) Patients with acute coronary syndrome confirmed by examination and chest pain caused by rheumatic heart disease, cardiomegaly, severe neurosis, climacteric syndrome, hyperthyroidism, cervical spondylosis, choledochocardial syndrome, gastroesophageal reflux, hiatal hernia, aortic dissection, and so on; (14) History of specific bleeding or 
bleeding from warfarin; (15) Patients with previous hematopoietic diseases; (16) Patients who have undergone surgery within 4 weeks before beginning the study and were prone to bleeding (without PCl); (17) Patients who participated in other clinical studies or took investigational drugs for within 90 days; (18) Patients with known or suspected to be allergic to the drug in this study or allergic constitution; (19) Drug abusers. (patients with a recent history (in the recent 2 years) of alcohol abuse or a known history of drug dependence; (20) Psychopath; (21) Patients who have been judged ineligible by physicians for inclusion in the study; (22) Patients who were medical staff's family members or relatives.

\section{Criteria For Losing Cases}

In the case of quitting the study or losing the follow-up, the investigators should take active measures to complete the final test, in order to analyze efficacy and safety of treatment. All cases who missed the chance of full participation in the study were recorded in a case report form (CRF) and in research conclusion form and the reason of losing the study was noted. Participants who did not complete the research for the following reasons were taken eligible into consideration: Investigators decided to quit: (1) In case of allergic reaction or serious adverse events (AEs), the investigation prohibited the cases from following the study according to physicians' judgment; (2) Other complications and special physiological changes that occurred during the study, who did not further participate in the study; (3) Poor patients' compliance; Subjects who left the study by themselves: (1) Regardless of the reason, a patient would be unwilling or impossible to continue the clinical study, or recommended to withdraw the study by physicians; (2) Although a subject did not explicitly withdraw from the study, he/she may not accept the medicine or test, leading to loss of the study.

\section{Exclusion Criteria}

Participants who do not complete the research project for the following reasons were excluded: (1) Those who failed to meet the inclusion criteria and were incorrectly included or met any of the exclusion criteria; (2) Those who met the inclusion criteria, while have not used medicine after inclusion, or had no further consultation records; (3) Patients who changed the medicine or used combination therapy different from original prescription in the middle of the course by themselves, especially those who used the combination therapy influencing effectiveness and safety of the therapy regimen.

\section{Discontinuation criteria}

The purpose of discontinuation is to protect rights and interests of subjects, ensure quality of study, and avoid unnecessary economic losses. Criteria for discontinuation were as follows: (1) In case of serious safety problems in the study, the study was discontinued in-time; (2) If the medicine was found to have no clinical value in the study, the study was discontinued to avoid ineffective treatment for subjects and unnecessary economic loss; (3) Clinical study regimen has been found with major issues during study, thereby causing difficulty in evaluating the therapeutic effect; or a well-designed regimen with important deviations during implementation, causing difficulty in assessment of the therapeutic effect if continues; (4) The asking discontinuation on behalf of principal investigator's requests; (5) The China Food and Drug Administration (CFDA) recommended to discontinue the study for some reasons. 


\section{Recruitment strategies}

In order to recruit patients, advertisements were placed in a broad range of media outlets, including flyers within the hospital, as well as the Chinese Clinical Trial Registry website. Patients who were interested in the trial received information about the study. Each potential participant was informed that the participation is fully voluntary and refusal to participate in the research has no negative effect on their treatment. Those who would like to join the study were further assessed to determine whether they could meet the inclusion criteria or not.

\section{Randomization And Blinding}

In the present study, "interactive web-based response system (IWRS)" was employed for randomized allocation. The Department of Medical Statistics in the National Center for Cardiovascular Diseases (Beijing, China) was in charge of preparation and maintenance of the random system. For patients who met the inclusion and exclusion criteria, their medical data were collected, and relevant operation processes were automatically retained. The research team members, except for the clinical research methodology personnel, were blinded to the treatment and the group assignment. Patients were informed about the experimental group and the control group, while they were not informed about group assignment. Both YMOS and placebo were provided by the Qionglai Tianyin Pharmaceutical Co., Ltd. (Qionglai, China), and the preparation of placebo oral solution with the same appearance, similar color, smell and taste, as YMOS package was blinded to investigators, patients, and statistical experts. The study code was not revealed until the end of the study, unless a serious adverse event (AE) was reported.

\section{Intervention}

All the subjects included in the current study were randomly divided into experimental group and control group by researcher, received standardized treatment [23] + YMOS and standardized treatment + placebo (10 $\mathrm{ml}$ for three times/day for 12 weeks). It was found highly essential to take other medicines at an interval of more than $1 \mathrm{~h}$. According to the status of undergoing standardized treatment in the past, stratified random sampling was carried out. Previously, patients who were treated in accordance with standardization treatment for more than 1 week were directly assigned to the group for screening and randomized distribution. Patients who have not been previously treated in accordance with the standardized treatment plan were treated in accordance with the standardized treatment plan for 1 week (introduction period), and then enrolled for screening and randomized distribution. When a patient had an angina pectoris, one tablet $(0.5 \mathrm{mg})$ of nitroglycerin was taken under the tongue, and the information of the patient's diary card was recorded. One tablet could be repeated every $5 \mathrm{~min}$ for each episode until the pain eased. If the pain persists after accumulating 3 tablets for within $15 \mathrm{~min}$, it is essential to seek for medical attention immediately. Both placebo and YMOS may be dispensed by the Central Hospital Pharmacy as a set of boxes at the beginning of each study week. Patients were asked to return the boxes for checking patient compliance. The patients' treatment prescriptions and conditions were recorded in the CRF. Details of the study procedures are given in Table 1. 
Table 1

Study procedures of the trial

\begin{tabular}{|c|c|c|c|c|c|c|c|}
\hline \multirow{2}{*}{$\begin{array}{l}\text { Study procedures } \\
\text { Time point }\end{array}$} & \multirow{2}{*}{$\begin{array}{l}\text { Lead-in } \\
\text { period } \\
-1 w \pm 2 d\end{array}$} & \multicolumn{4}{|c|}{ Treatment period } & \multicolumn{2}{|c|}{ Follow-up period } \\
\hline & & 0 & $4 w \pm 4 d$ & $8 w \pm 4 d$ & $12 w \pm 4 d$ & $16 w \pm 6 d$ & $20 w \pm 6 d$ \\
\hline Clinical data & $\sqrt{ }$ & & & & & & \\
\hline $\begin{array}{l}\text { Combined use of } \\
\text { drugs }\end{array}$ & $\sqrt{ }$ & & $\sqrt{ }$ & $\sqrt{ }$ & $\sqrt{ }$ & $\sqrt{ }$ & $\sqrt{ }$ \\
\hline Medical examination & $\sqrt{ }$ & $\sqrt{ }$ & $\sqrt{ }$ & $\sqrt{ }$ & $\sqrt{ }$ & $\sqrt{ }$ & $\sqrt{ }$ \\
\hline \multicolumn{8}{|l|}{$\begin{array}{l}\text { Therapeutic } \\
\text { evaluation }\end{array}$} \\
\hline SAQ & $\sqrt{ }$ & & $\sqrt{ }$ & $\sqrt{ }$ & $\sqrt{ }$ & $\sqrt{ }$ & $\sqrt{ }$ \\
\hline $\operatorname{ccs}$ & $\sqrt{ }$ & & $\sqrt{ }$ & $\sqrt{ }$ & $\sqrt{ }$ & $\sqrt{ }$ & $\sqrt{ }$ \\
\hline NYHA & $\sqrt{ }$ & & $\sqrt{ }$ & $\sqrt{ }$ & $\sqrt{ }$ & $\sqrt{ }$ & $\sqrt{ }$ \\
\hline VAS & $\sqrt{ }$ & & $\sqrt{ }$ & $\sqrt{ }$ & $\sqrt{ }$ & $\sqrt{ }$ & $\sqrt{ }$ \\
\hline HAMD & $\sqrt{ }$ & & $\sqrt{ }$ & $\sqrt{ }$ & $\sqrt{ }$ & $\sqrt{ }$ & $\sqrt{ }$ \\
\hline HAMA & $\sqrt{ }$ & & $\sqrt{ }$ & $\sqrt{ }$ & $\sqrt{ }$ & $\sqrt{ }$ & $\sqrt{ }$ \\
\hline $\begin{array}{l}\text { Weekly incidence of } \\
\text { angina }\end{array}$ & $\sqrt{ }$ & & $\sqrt{ }$ & $\sqrt{ }$ & $\sqrt{ }$ & $\sqrt{ }$ & $\sqrt{ }$ \\
\hline $\begin{array}{l}\text { Nitroglycerin stopping } \\
\text { rate }\end{array}$ & & & $\sqrt{ }$ & $\sqrt{ }$ & $\sqrt{ }$ & $\sqrt{ }$ & $\sqrt{ }$ \\
\hline SF-12 & $\sqrt{ }$ & & $\sqrt{ }$ & $\sqrt{ }$ & $\sqrt{ }$ & $\sqrt{ }$ & $\sqrt{ }$ \\
\hline \multicolumn{8}{|l|}{ Safety indicators } \\
\hline $\begin{array}{l}\text { Blood routine } \\
\text { examination }\end{array}$ & $\sqrt{ }$ & & & & $\sqrt{ }$ & & \\
\hline $\begin{array}{l}\text { Urine and stool routine } \\
\text { examination }\end{array}$ & $\sqrt{ }$ & & & & $\sqrt{ }$ & & \\
\hline $\begin{array}{l}\text { Hepatic and renal } \\
\text { function }\end{array}$ & $\sqrt{ }$ & & & & $\sqrt{ }$ & & \\
\hline $\begin{array}{l}\text { Fasting blood glucose } \\
\text { and blood lipid }\end{array}$ & $\sqrt{ }$ & & & & $\sqrt{ }$ & & \\
\hline
\end{tabular}




\begin{tabular}{|c|c|c|c|c|c|c|}
\hline Coagulation & $\sqrt{ }$ & & & $\sqrt{ }$ & & \\
\hline Troponin Tnl & $\sqrt{ }$ & & & $\sqrt{ }$ & & \\
\hline $\begin{array}{l}\text { Electrocardiogram } \\
\text { examination }\end{array}$ & $\sqrt{ }$ & & & $\sqrt{ }$ & & \\
\hline $\begin{array}{l}\text { Thyroid function } \\
\text { examination (when } \\
\text { necessary) }\end{array}$ & $\sqrt{ }$ & & & $\sqrt{ }$ & & \\
\hline $\begin{array}{l}\text { Adverse events } \\
\text { records }\end{array}$ & & $\sqrt{ }$ & $\sqrt{ }$ & $\sqrt{ }$ & $\sqrt{ }$ & $\sqrt{ }$ \\
\hline \multicolumn{7}{|l|}{ Other procedures } \\
\hline \multicolumn{2}{|l|}{ Random grouping } & \multicolumn{3}{|l|}{$\sqrt{ }$} & & \\
\hline \multicolumn{2}{|l|}{$\begin{array}{l}\text { Distribute research } \\
\text { drugs }\end{array}$} & $\sqrt{ } \sqrt{ }$ & \multicolumn{2}{|l|}{$\sqrt{ }$} & & \\
\hline Distribute nitroglycerin & $\sqrt{ }$ & $\sqrt{ } \sqrt{ }$ & $\sqrt{ }$ & $\sqrt{ }$ & $\sqrt{ }$ & \\
\hline $\begin{array}{l}\text { Issue patient diary } \\
\text { card }\end{array}$ & $\sqrt{ }$ & $\sqrt{ } \sqrt{ }$ & $\sqrt{ }$ & $\sqrt{ }$ & $\sqrt{ }$ & \\
\hline \multicolumn{2}{|l|}{$\begin{array}{l}\text { Recycling of } \\
\text { remaining research } \\
\text { drugs and kit } \\
\text { packaging }\end{array}$} & $\sqrt{ }$ & $\sqrt{ }$ & $\sqrt{ }$ & $\sqrt{ }$ & $\sqrt{ }$ \\
\hline \multicolumn{2}{|l|}{$\begin{array}{l}\text { Recovery of remaining } \\
\text { nitroglycerin }\end{array}$} & $\sqrt{ } \sqrt{ }$ & $\sqrt{ }$ & $\sqrt{ }$ & $\sqrt{ }$ & $\sqrt{ }$ \\
\hline \multicolumn{2}{|l|}{$\begin{array}{l}\text { Recovery of patient } \\
\text { diary card }\end{array}$} & $\sqrt{ } \sqrt{ }$ & $\sqrt{ }$ & $\sqrt{ }$ & $\sqrt{ }$ & $\sqrt{ }$ \\
\hline \multicolumn{2}{|l|}{$\begin{array}{l}\text { Clinical efficacy } \\
\text { judgment }\end{array}$} & & & $\sqrt{ }$ & & $\sqrt{ }$ \\
\hline \multicolumn{7}{|c|}{$\begin{array}{l}\text { Note: SAQ Seattle angina questionnaire, HAMA Hamilton anxiety scale, HAMD Hamilton depression } \\
\text { scale, CCS Canadian Cardiovascular Society, NYHA New York Heart Association classification, VAS } \\
\text { visual analogue scale. }\end{array}$} \\
\hline
\end{tabular}

The standardized treatment plan must include the following 3 types of drugs (the use of drugs was recorded in the combination mode of medical records): (1) Anti-platelet aggregation drugs: aspirin (75$100 \mathrm{mg}, \mathrm{QD})$, patients, who could not tolerate aspirin, could receive clopidogrel as an alternative therapy. Patients with a previous history of $\mathrm{PCl}$ surgery simultaneously received both drugs. (2) Lipid-lowering drugs: statins, such as Attovastatin (10-20 mg, QD) and simvastatin (20-40 mg, QD). (3) Anti-ischemic medications: $\triangle$ $ß$-receptor blockers: metoprolol sustained-release tablets (50-200 mg QD) were 
recommended; $\varangle$ Similar stable dose of receptor blockers; 『Long-acting nitrates, involving isosorbide mononitrate sustained-release tablets (40-60 mg QD); Calcium antagonists (e.g., amlodipine tablets (5$10 \mathrm{mg}$ QD). In addition, patients with diabetes or hypertension were recommended to take angiotensinconverting enzyme (ACE) inhibitors or angiotensin-receptor blockers (ARBs) as secondary prophylaxis drugs for stable angina pectoris, including lisinopril (10-20 mg QD) or losartan (50mg QD). Drugs necessary for other diseases could be additionally used, while the record form of combined therapy was filled out. During the trial, it was prohibited to use Western medicines for the treatment of chronic stable angina pectoris except for standardized treatment and nitroglycerin. The combined use of other proprietary Chinese medicines and traditional Chinese medicine was also prohibited during the trial.

\section{Outcome Measures}

At baseline (prior to starting either intervention), every 4 weeks after the intervention and every 4 weeks in the follow-up, all the patients were asked to complete SAQ, HAMA, HAMD, CCS, NYHA, VAS, and SF-12, while the frequency of angina-related symptoms per week and the number of nitroglycerin use were recorded in the patient diary card. Besides, all the patients underwent laboratory examinations at baseline and at the 4th week after the intervention. During the follow-up, any registered participants who could not continue the study during the treatment period remained in their randomized group for intention-to-treat (ITT) analysis.

\section{Primary outcomes}

The AF in SAQ for 12 weeks was taken as the primary outcome.

\section{Key Secondary Outcome}

Changes in HAMA score compared with baseline during 12-week follow-up were noted.

\section{Secondary outcomes}

Secondary outcomes were as follows: (1) Reduction rate of HAMA scale score at the 12th week (reduction rate of HAMA score $=[$ (pre-treatment score - post-treatment score $) /$ pre-treatment score $] \mathrm{x}$ $100 \%$ ). A reduction rate of HAMA score $>75 \%$ is clinically cure; a reduction rate of $50-75 \%$ is markedly effective; a reduction rate of $25-49 \%$ is effective; a reduction rate of $<25 \%$ is invalid; (2) CCS and NYHA grades; (3) VAS score; (4) HAMD score; (5) AF of weekly angina; (6) Dosage of nitroglycerin; (7) SF-12 score. (8) PL, AS, TS, and DP scores in SAQ.

\section{Safety examination indices}

Safety examination indices were as follows: (1) New vascular events; (2) All-cause mortality events; (3) Severe bleeding events (as defined by Global Strategies for Opening Occluded Coronary Arteries (GUSTO) classification); (4) Moderate bleeding events (as defined by GUSTO); (5) Hepatic and renal functions; (6) $\mathrm{AEs} /$ serious AEs reported by investigators: any possible adverse reactions/AEs that were observed during 
clinical study, and recorded truthfully, such as fever, blush, rash, pruritus, diarrhea, dizziness, headache, etc. Pay special attention to the observation and record drug allergy and local irritation (Table 2).

Table 2

Primary outcomes, secondary outcomes and safety examination indices

\begin{tabular}{|c|c|}
\hline \multirow{2}{*}{$\begin{array}{l}\text { Primary } \\
\text { outcomes }\end{array}$} & (1) SAQ Score \\
\hline & (2) HAMA score changes relative to baseline at 12 weeks of follow-up \\
\hline \multirow{7}{*}{$\begin{array}{l}\text { Secondary } \\
\text { outcomes }\end{array}$} & (1) HAMA scale at the 12th week Score reduction rate \\
\hline & (2) CCS and NYHA grades \\
\hline & (3) Visual analogue scale \\
\hline & (4) HAMD \\
\hline & (5) Attack frequency of weekly angina \\
\hline & (6) Dosage of nitroglycerin \\
\hline & (7) SF-12 Quality of Life Scale \\
\hline \multirow{6}{*}{$\begin{array}{l}\text { Safety } \\
\text { examination } \\
\text { indices }\end{array}$} & (1) New vascular events \\
\hline & (2) All-cause mortality events \\
\hline & (3) Severe bleeding events (as defined by GUSTO) \\
\hline & (4) Moderate bleeding events (as defined by GUSTO) \\
\hline & (5) Hepatic and renal function evaluation \\
\hline & $\begin{array}{l}\text { (6) Adverse events/serious adverse events reported by investigators: any possible } \\
\text { adverse reactions/adverse events are closely observed during clinical study, and } \\
\text { recorded truthfully, such as fever, blush, rash, pruritus, diarrhea, dizziness, headache, } \\
\text { etc. Pay special attention to the observation and record drug allergy and local } \\
\text { irritation. }\end{array}$ \\
\hline
\end{tabular}

Note: SAQ Seattle angina questionnaire, HAMA Hamilton anxiety scale, HAMD Hamilton depression scale, CCS Canadian Cardiovascular Society, NYHA New York Heart Association classification, VAS visual analogue scale.

\section{Follow-up protocol}

The patients were followed-up at the 4th and 8th weeks after the treatment, and they had to complete the above-mentioned questionnaire and records. Researchers assessed patients' health condition by inspecting the medical records, which acquired completing the CRF.

\section{Adverse events (AEs)}

Although no research has still reported AEs of YMOS, all drugs may have side effects or allergic reactions. Any discomfort or unexpected conditions that occurred during the experiment were taken as 
AEs into consideration, regardless of whether they were related to research interventions. All the AEs were recorded in detail in the CRF. Serious AEs were immediately reported to the principal investigator, including death, life-threatening or severe or permanent disability. The Ethics Committee assessed whether the AEs were associated with the experimental drugs.

\section{Data management}

To ensure strict adherence to the research protocol and familiarity with the trial management process, the main research members attempted to establish an independent committee in the beginning of the study. Data management personnel of the committee were qualified, received effective training, and were familiar with the functions of data management. The data management of this clinical trial, including the confirmation of all subjects (being able to effectively check different records, such as medical records and hospital records), all the signed written informed consent forms, all medical records, detailed records related to drug distribution, was conducted. When the patients were recruited into research project, researchers collected their demographic and baseline characteristics by using standard CRF. Data were first processed. The results of questionnaires, clinical outcomes, AEs, and the reasons for the patients' withdrawal from the study were detailed in the CRFs. To reduce errors, CRF data were assessed independently by two researchers. They checked each other's input values, and only consistent data were stored in the database. Paper files were kept in a locked file cabinet in the hospital, while electronic files, laboratory test results, were stored on a password-protected computer and could only be reached by the principal investigator.

\section{Determination of the sample size}

The present study was designed as a prospective, multicenter, randomized, double-blind, placebocontrolled trial to verify the therapeutic effects of YMOS on patients with chest pain. The calculation of sample size firstly aimed to main evaluation outcomes, including actual SAQ scores at follow-up (for 12week). Based on the results of previous research and related literature, it was assumed that SAQ score in the experimental group was increased by at least 10 points compared with that in the control group, and the standard deviation (SD) of SAQ score was conservatively estimated to be \pm 25 . Besides, 320 patients were scheduled to be included in the trial on the premise that the sample size was $5 \%$ on both sides of the statistical significance level and $10 \%$ off rate was taken into account. Additionally, $90 \%$ assurance was provided to prove that the effects in the experimental group were superior than those in the control group.

Moreover, according to the literature and clinical experience, it was estimated that the difference between the experimental group and the control group in HAMA score was equal to 1 (the average HAMA score in the experimental group was 1 point lower than that in the control group), and the SD of changing the score point was conservatively estimated to be \pm 3 . The significance level of the study test was $5 \%$ on both sides. Considering $10 \%$ drop off rate, $80 \%$ assurance rate was provided to prove that the experimental group outperformed the control group in the reduction of HAMA score.

Overall, to meet the evaluation needs of both the main outcomes and the key secondary outcomes, the enrollment size of the study was finally set to 320 cases, who were randomly assigned to two groups at 
ratio of $1: 1$ ( $n=160$ patients for each group).

\section{Statistical analysis}

The statistical analysis was conducted in accordance with the principle of ITT. All randomized patients were included in the final analysis (whether against the protocol or not), and the key efficacy outcomes were simultaneously assessed on the basis of the population's compliance with the therapeutic plan.

For descriptive analysis, quantitative indices were expressed as mean $\pm S D$, and qualitative indices were presented as frequency and/or percentage. For making inter-group comparison, a parametric or a nonparametric method was employed for analysis of the quantitative indices according to their normality status, while chi-square or the Fisher's exact test was used for qualitative indices based on the expected frequency.

For the analysis of ou, the analysis of covariance (ANCOVA) model adjusted for baseline and central effects was utilized to calculate the difference in the score between the two groups (or change in the score relative to baseline), and corresponding $95 \%$ confidence interval $(95 \% \mathrm{Cl})$ was given. The secondary outcomes were analyzed by using the same statistical analysis method. Significance level for all statistical tests was $5 \%$, and statistical analysis was undertaken by using SAS 9.4 software (SAS Institute, Cary, NC, USA).

\section{Discussion}

Although $\mathrm{PCl}$ is highly advantageous for patients with CHD, PPCP remains a main challenge. Chinese herbs have shown their significant therapeutic effects for patients with CHD and depression [24, 25]. Besides, YMOS has been found advantageous for patients with chronic stable angina [13-15]. In the current study, we attempted to perform a multicenter study, while expanded the sample size to further confirm the efficacy and safety of YMOS for PPCP patients. We used AF in SAQ as the primary outcome and changes in HAMA as the key secondary outcome to evaluate the efficacy of YMOS. In the present research, in order to further reveal whether YMOS could improve the symptoms of PPCP patients, in addition to the SAQ scale, psychological scales were included and analyzed.

There are a number of limitations in this study. Firstly, although we recruited patients with PPCP from multiple centers, however, the sample size was small, and further research with larger sample size needs to be conducted. Secondly, a larger sample size and multi-center study may result in different achievements for the calculation of sample size, which can be attributed to pilot-based nature of the study and clinical observations.

In summary, our findings may provide a solid foundation for the clinical treatment of patients with PPCP, and present further evidence for the treatment of PPCP patients using traditional Chinese medicine.

\section{Trial Status}


This research(version 4.0, March 31, 2020) has been registered at the Chinese Clinical Trial Registry (Registration No. ChiCTR2000028760). The recruitment began at May 7, 2020 and will be completed on January 1, 2025.

\section{Declarations}

\section{Acknowledgements}

Not applicable.

\section{Authors' contributions}

DPX and YJW planned the protocol and revised the manuscript. BXW participated in designing the trial and drafted the manuscript. JHL and ZKY recruited and screened eligible patients in the inpatient department. HYX and LQL was responsible for generating and distributing the random numbers. QRL and BYZ participated in designing the outcome measurements and assessing the outcomes. YND participated in assessing treatment efficacy and collected clinical data. ZWH was the study coordinator. MYL analyzed the clinical data. All the authors have read and approved the final manuscript.

\section{Funding}

This project was funded by Qionglai Tianyin Pharmaceutical Co., Ltd. by providing the subjects' inspection costs in the research. Funder will have ultimate authority over any activities including study design; collection, management, analysis, and interpretation of data; writing of the report; and the decision to submit the report for publication.

\section{Availability of data and materials}

The trial results will be published in a peer-reviewed scientific paper and through oral presentations at conferences. The datasets analysed during the current study are available from the corresponding author on reasonable request.

\section{Ethics approval and consent to participate}

This study was approved by the Ethics Committee of the Guangdong Provincial Hospital of Traditional Chinese Medicine (Guangzhou, China) (Approval No. BF2020-025-01), and we will not begin recruiting at other centers in the trial until local ethical approval has been obtained. All patients were informed about the risks and benefits of the treatment strategy and signed informed consent forms at the beginning of the study.

\section{Consent for publication}

Not applicable. 


\section{Competing interests}

The authors declare that they have no competing interests

\section{Author details}

${ }^{1}$ Cardiac Disease Treatment Center, Guangdong Hospital of Traditional Chinese Medicine, Guangdong, China

${ }^{2}$ The Second Clinical Medical College, Guangzhou University of Traditional Chinese Medicine, Guangdon, China

${ }^{3}$ Department of Cardiovascular Disease Center, Xiyuan Hospital, China Academy of Chinese Medical Sciences, Beijing100091, China

${ }^{4}$ Fuwai Hospital, National Center for cardiovascular Disease 『Beijing100091

${ }^{5}$ Department of Cardiology, Fuwai Hospital, National Center for Cardiovascular Disease, Chinese Academy of Medical Sciences and Peking Union Medical College

\section{References}

1. Joseph, D. Leong, M. McKee, S.S. Anand, J.D. Schwalm, K. Teo, A. Mente, S. Yusuf, Reducing the Global Burden of Cardiovascular Disease, Part 1: The Epidemiology and Risk Factors, Circ Res, 121 (2017) 677-694.

2. Zhou, H. Wang, X. Zeng, P. Yin, J. Zhu, W. Chen, X. Li, L. Wang, L. Wang, Y. Liu, J. Liu, M. Zhang, J. Qi, S. Yu, A. Afshin, E. Gakidou, S. Glenn, V.S. Krish, M.K. Miller-Petrie, W.C. Mountjoy-Venning, E.C. Mullany, S.B. Redford, H. Liu, M. Naghavi, S.I. Hay, L. Wang, C.J.L. Murray, X. Liang, Mortality, morbidity, and risk factors in China and its provinces, 1990-2017: a systematic analysis for the Global Burden of Disease Study 2017, Lancet, 394 (2019) 1145-1158.

3. Gruntzig, Transluminal dilatation of coronary-artery stenosis, Lancet, 1 (1978) 263.

4. L. Bhatt, Percutaneous Coronary Intervention in 2018, JAMA, 319 (2018) 2127-2128.

5. R. Kandan, T.W. Johnson, Management of percutaneous coronary intervention complications, Heart, 105 (2019) 75-86.

6. J. Anderson, Chest Pain After Percutaneous Coronary Intervention: More Than Meets the Eye, Can J Cardiol, 31 (2015) 960-962.

7. H. Hofma, W.J. van der Giessen, B.M. van Dalen, P.A. Lemos, E.P. McFadden, G. Sianos, J.M. Ligthart, D. van Essen, P.J. de Feyter, P.W. Serruys, Indication of long-term endothelial dysfunction after sirolimus-eluting stent implantation, Eur Heart J, 27 (2006) 166-170.

8. Ong, A. Athanasiadis, A. Perne, H. Mahrholdt, T. Schaufele, S. Hill, U. Sechtem, Coronary vasomotor abnormalities in patients with stable angina after successful stent implantation but without in-stent 
restenosis, Clin Res Cardiol, 103 (2014) 11-19.

9. Li, D. Yang, L. Lu, D. Wu, J. Yao, X. Hu, M. Long, C. Luo, Z. Du, Thermodilutional Confirmation of Coronary Microvascular Dysfunction in Patients With Recurrent Angina After Successful Percutaneous Coronary Intervention, Can J Cardiol, 31 (2015) 989-997.

10. S. Hayek, Y.A. Ko, M. Awad, A. Del Mar Soto, H. Ahmed, K. Patel, M. Yuan, S. Maddox, B. Gray, J. Hajjari, L. Sperling, A. Shah, V. Vaccarino, A.A. Quyyumi, Depression and chest pain in patients with coronary artery disease, Int J Cardiol, 230 (2017) 420-426.

11. M. Pardue, K.S. White, E.V. Gervino, The Role of Disease Conviction: Exploring Its Effects on Chest Pain and Anxiety-Related Models of Non-cardiac Chest Pain, J Clin Psychol Med Settings, 26 (2019) 131-141.

12. Loerbroks, J.A. Bosch, P.M. Mommersteeg, R.M. Herr, P. Angerer, J. Li, The association of depression and angina pectoris across 47 countries: findings from the 2002 World Health Survey, Eur J Epidemiol, 29 (2014) 507-515.

13. C. Huffman, C.M. Celano, S.R. Beach, S.R. Motiwala, J.L. Januzzi, Depression and cardiac disease: epidemiology, mechanisms, and diagnosis, Cardiovasc Psychiatry Neurol, 2013 (2013) 695925.

14. Li GX. Yinxing Mihuan oral solution for the treatment of coronary atherosclerotic heart disease angina pectoris: a case report of 60 cases. Henan Traditional Chinese Medicine. 2013,33(6):887-888

15. Peng LL, Gong JX. Observation on the effect of Yinxing Mihuan oral solution in the treatment of coronary heart disease angina pectoris. Journal of Sichuan of Traditional Chinese Medicine. 2017,35(4):192-193

16. Yan J, Yuan S. Clinical observation of Ginkgo Leaf Extract and Armillariella Mellea Powders Oral Solution combined with Aspirin Enteric-coated Tablets and clopidogrel in treatment of coronary heart disease with angina pectoris. Drugs and Clinic.2016,31(10):1575-1578

17. Chen Q, A case of skin reaction caused by Yinxing Mihuan oral solution, Shanxi Journal of Traditional Chinese Medicine, 2013,29(10):62.

18. Chen, M. Hei, L. Kong, Y. Liu, Y. Yang, H. Mu, X. Zhang, S. Zhao, J. Duan, One water-soluble polysaccharide from Ginkgo biloba leaves with antidepressant activities via modulation of the gut microbiome, Food Funct, 10 (2019) 8161-8171.

19. Woelk, K.H. Arnoldt, M. Kieser, R. Hoerr, Ginkgo biloba special extract EGb 761 in generalized anxiety disorder and adjustment disorder with anxious mood: a randomized, double-blind, placebo-controlled trial, J Psychiatr Res, 41 (2007) 472-480.

20. I. Gavrilova, U.W. Preuss, J.W. Wong, R. Hoerr, R. Kaschel, N. Bachinskaya, G.I.S. Group, Efficacy and safety of Ginkgo biloba extract EGb 761 in mild cognitive impairment with neuropsychiatric symptoms: a randomized, placebo-controlled, double-blind, multi-center trial, Int J Geriatr Psychiatry, 29 (2014) 1087-1095.

21. Singh SK, Barreto GE, Aliev G, Echeverria V.Ginkgo biloba as an Alternative Medicine in the Treatment of Anxiety in Dementia and other Psychiatric Disorders.Curr Drug Metab. 2017;18(2):112-119. 
22. Zhou LS. Clinical observation of armillaria miltiorrhiflora tablets in treatment of shensensenility, hypertension, coronary heart disease, vertigo syndrome and other 150 cases, Journal of chengdu college of traditional Chinese medicine, 1980,(4):37-41

23. Gao RL. Guidelines for the diagnosis and treatment of chronic stable angina. Chinese Journal of Cardiovascular Diseases, 2007, 35(3): 195-206

24. Wang, Y.H. Shi, Z. Xu, H. Fu, H. Zeng, G.Q. Zheng, Efficacy and safety of Chinese herbal medicine for depression: A systematic review and meta-analysis of randomized controlled trials, J Psychiatr Res, 117 (2019) 74-91.

25. Yuan, W.L. Shi, Q.Q. Xin, K.J. Chen, W.H. Cong, Holistic Regulation of Angiogenesis with Chinese Herbal Medicines as a New Option for Coronary Artery Disease, Evid Based Complement Alternat Med, 2018 (2018) 3725962.

\section{Figures}



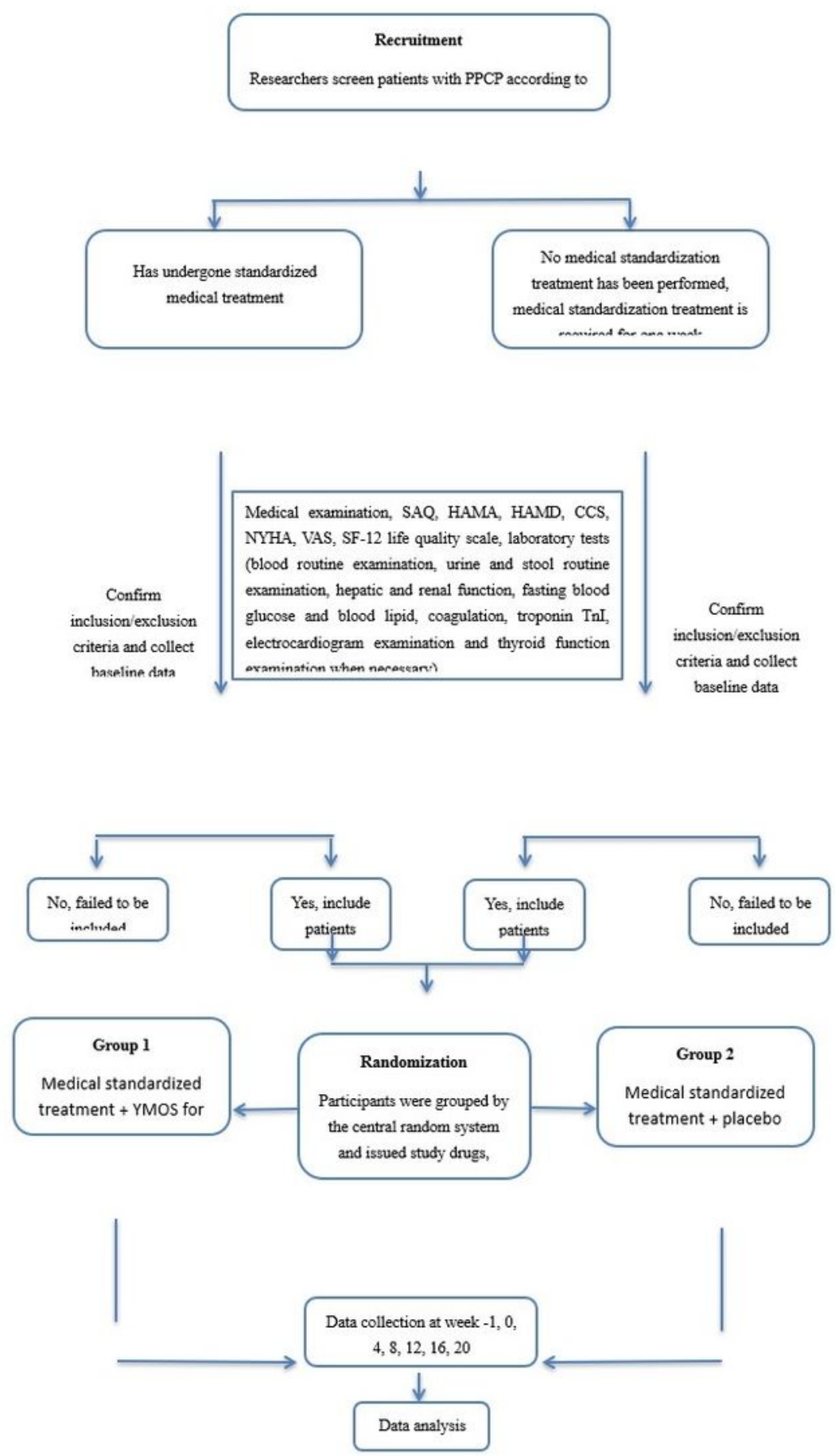

\section{Figure 1}

Flowchart of the trial Note: SAQ Seattle angina questionnaire, HAMA Hamilton anxiety scale, HAMD Hamilton depression scale, CCS Canadian Cardiovascular Society, NYHA New York Heart Association classification, VAS visual analogue scale.

\section{Supplementary Files}


This is a list of supplementary files associated with this preprint. Click to download.

- InformationLeafletforlnformedConsent.doc

- SPIRITChecklist.doc 